\title{
Return, purchase, or skip? Outcome, duration, and consumer behavior in the rent-to-own market
}

\author{
Michael H. Anderson • Sanjiv Jaggia
}

\begin{abstract}
Rent-to-own (RTO) is attractive to financially distressed consumers. It allows immediate access to merchandise and an opportunity for eventually acquisition. Yet goods can be returned at any point without penalty or other adverse consequences. We use a competing risk methodology that accounts for unobserved consumer heterogeneity to study how contracts conclude, estimating the probabilities of exitvia return, purchase, or skip - and the associated durations. The estimated outcome probabilities highlight the use of the embedded return option by RTO consumers and the trade-offs and cross-subsidization implicit in the RTO contractual arrangement. We offer rational and behavioral explanations of consumer behavior in the RTO market, which we believe can be generalized to other consumer loan markets.
\end{abstract}

Keywords Competing risks - Unobserved heterogeneity - Transaction data . Consumer credit $\cdot$ Payment schedule $\cdot$ Sub-prime lending

JEL Classification $\quad \mathrm{C} 24 \cdot \mathrm{D} 14 \cdot \mathrm{G} 29$

\section{Introduction}

This article studies the nature of rent-to-own (RTO) transactions. Since its beginnings in the 1960s, the RTO industry has grown to over 8,000 stores in the US, 
annually serving over 3 million customers, generating over $\$ 5$ billion in revenue (APRO 2001). The clientele consists primarily of disadvantaged consumers having restricted access to alternative (conventional) financing mechanisms such as credit cards or bank loans. ${ }^{1}$ The RTO transaction allows a customer immediate access to new or used merchandise-most commonly appliances, electronics, or furniturewith neither a credit check nor down payment in exchange for a rental payment due either weekly, bi-weekly, or monthly. Delivery, set up, and service are typically also bundled in the contract. The agreement has a fixed contractual term, usually from 12 to 24 months; and, by making all payments, or exercising an early purchase option, the consumer acquires the merchandise. At the same time, goods can be returned, at any point, without penalty or other adverse consequences. The option to rent or to purchase thus remains with the customer over the life of the contract. How this contract is actually used is interesting, in and of itself, and has been a subject of debate among consumer advocates and others. ${ }^{2}$ Despite the importance of these issues, basic questions have been answered only anecdotally and definitive empirical stylized facts are not available.

The nature of the RTO transaction - rental or purchase agreement—is a key policy question. The industry is pursuing national legislation classifying it as a lease, while some states have classified it as an installment credit agreement. Clearly, if it is most appropriately described as a purchase then this transaction is usurious. This is partly an empirical question judged by how the contract is actually being used by consumers, e.g., the probability of purchase and the expected duration of the agreement. In this article, we analyze a unique data set of some 7,500 detailed transactional records to better understand RTO usage. We employ a multiple destination (competing risk) framework to explore how and when a given contract concludes, where conclusion occurs in one of three ways (exits): return, purchase, or skip. In particular, "return" means payments cease and the merchandise is returned to the store, perhaps involuntarily. "Purchase" means ownership is transferred to the customer, possibly through the exercise of an early purchase option. "Skip," also referred to as a "default" or "write off," is when payments prematurely stop but the merchandise cannot be recovered by the store for some reason-"the customer skipped with it."

It is well documented that the primary market for RTO is the "working poor." Along with other subprime lenders-e.g., payday lenders and pawn shops-RTO functions, in part, to provide credit for those who are otherwise not credit-worthy. One major difference is that a RTO agreement has several embedded options. First and foremost, the agreement carries the right of return. This is, at any point, at the customer's instigation, the merchandise can be return to the store and the agreement terminated. This is valuable for a customer who has only a short term need or, more importantly,

\footnotetext{
${ }^{1}$ For example, the FTC in their survey of RTO customers (Federal Trade Commission 2000) report that among consumers using RTO in the last year 56.3\% (36.1\%) did not have a credit card (checking account) versus a national rate of $33.5 \%$ (15.0\%).

2 For example, Freedman (1993) notes the high annual percentage interest rates and Walden (1990) argues that RTO prices substantially exceed dealer cost, while Hill et al. (1998) argue that prices cannot be justified by the return option or other services offered. Anderson and Jackson (2001), Swagler and Wheeler (1989), and Zikmund-Fisher and Parker (1999) examine consumer experience and motivation for entering into a RTO agreement.
} 
uncertainty over the precise length of need. It also makes the transaction a rental with no adverse credit impact, making the transaction well suited to someone facing uncertainty regarding his/her ability to make the required payments. Additionally, this option feature is potentially quite valuable as it allows one to sidestep the "credit trap" occurring with, say, payday lending (e.g., Stegman and Faris 2003). ${ }^{3}$ Second, the contract also offers the option to early purchase, i.e., to terminate the contract at any point and take ownership of the merchandise by paying a proportion of the remaining payments-typically about one half (see, e.g., Federal Trade Commission 2000). This is valuable for a consumer, with insufficient means to acquire desired merchandise, who experiences an improvement in fortune. Both of these embedded options explain why one would expect the typical length of a contract to be less than the contractual term set by the store.

In this article, we estimate the return, purchase, and skip terminal probabilities and the actual length of the contracts which together provide insight into the use of embedded options. Our analysis is aided by rich details of consumer transactions as opposed to other studies that are based on relatively small survey data which may be prone to misreporting. For instance, the well-documented study of RTO by the Federal Trade Commission (2000) surveyed 524 customers to produce primarily descriptive analysis. ${ }^{4}$ While our descriptive analysis of consumer demographics is consistent with theirs, the outcome variables are quite different. For instance, we find that only $23.6 \%$ of completed RTO contracts represent a purchase, which is starkly lower than $64.3 \%$ as reported by the FTC study.

The statistical model in this article accounts for both observed and unobserved sources of heterogeneity. Observed heterogeneity includes factors representing customer demographics as well as the structure of the contract. Regarding demographics, we expect a picture of the "working poor" to emerge. At the same time, it is not clear what will be the impact on the exit probabilities of such things as age or income, as theories could be formulated positing various relations to outcome. It is even more interesting to evaluate the impact of how the contracts are structured. For instance, in contrast to conventional financial arrangements, the customer can pick the payment frequency - weekly, bi-weekly, or monthly. The choice could depend, for instance, on the length of planned product use or to match one's paycheck schedule. ${ }^{5}$ Factors such as payment frequency and contractual term are chosen by customers based on an evaluation of their financial situation and needs. We believe that what they end up choosing will further dictate the contract evolution. For instance, the more frequent

\footnotetext{
3 This credit trap occurs because one can get access to future cash flows in exchange for a fixed fee. However, since such lending appeals to financially constrained borrowers, such loans frequently need to be "rolled over" with the payment of an additional fee which exacerbates the consumer's financial situation leading to further loan demand as well as an ever higher effective APR (Federal Deposit Insurance Corporation 2005).

${ }^{4}$ Lacko et al. (2002) and McKernan et al. (2003) further analyze the FTC data and include a nice regulatory overview. Anderson and Jaggia (2009) analyze transactional data; however, they do not explore the various outcomes of an RTO contract.

5 The payment is determined by the store by marking their product cost up 2 to 3 times (Federal Trade Commission 2000) then dividing that into equal monthly amounts, where the contractual term (number of payments) is chosen to make the resultant periodic payment attractive to customers. Subsequently, if weekly (bi-weekly) is selected the periodic payment will be one fourth (one half) of what would be due monthly.
} 
the payments, the more one's attention is focused on the contract, possibly resulting in quicker resolution. Similarly, consumers may adjust their decision time scale in accordance with the contractual term. These explanations tie in with the behavioral literature that suggests that consumers mentally track the cost and benefits of a transaction with symbolic linkage between consumption and the way the purchase is financed (see, e.g., Prelec and Loewenstein 1998; Thaler 1985).

Overall, the article contributes useful insights regarding behavior in the relatively unexplored RTO market. Apart from a detailed descriptive analysis of the transactional data, we estimate a competing risk model to better understand contract usage. The article also illustrates the trade-offs and cross-subsidization implicit in the RTO contractual arrangement. We compute effective interest rates for RTO customers which are significantly smaller than the generally reported APRs. Finally, we believe that our rational and behavioral explanations of consumer behavior in the RTO market can be generalized to other consumer loan markets.

The rest of the article is organized in the following way. The next section outlines the competing risk model methodology. Section 3 describes the sample characteristics of the data set and the variables used in the model. It also suggests a method to compute the annual percentage rates paid by RTO customers. Section 4 discusses the empirical results, identifying several empirical regularities. Section 5 concludes.

\section{Methodology}

Multiple destination, or competing risk, models have become increasingly popular in economics where the duration of an event is terminated by exit to one of several mutually exclusive and collectively exhaustive destinations-the competing risks (see Lancaster 1990 for an overview). These models have been used to model, e.g., unemployment, welfare spells, and mortgage terminations (see Katz and Meyer 1990; Blank 1989; Deng et al. 2000, respectively). In this article, we employ a competing risk model to explore when and how an RTO contract concludes. In other words, based on customer- and contract-specific variables (observed heterogeneity), the model predicts how long the contract lasts and whether it is terminated in a return, purchase, or skip. In addition to observed heterogeneity, the statistical model also accounts for unobserved characteristics of RTO customers. While the next section discusses the data in detail, we first want to note the main aspects of our model.

In our setting, we have three mutually exclusive and collectively exhaustive exits (contract conclusions). Let $T_{\mathrm{r}}, T_{\mathrm{p}}, T_{\mathrm{S}}$ be the latent variables that capture the length of time of an RTO contract until is terminated by the consumer via return, purchase, or skip, respectively. The observed data consist of information on $T=\min \left(T_{\mathrm{r}}, T_{\mathrm{p}}, T_{\mathrm{s}}\right)$ along with indicator variables, $I_{\mathrm{r}}, I_{\mathrm{p}}$, and $I_{\mathrm{s}} . T$ is the actual duration (length of time the contract actually ran) of an agreement and the indicator variables define the mode of exit. For instance, $I_{\mathrm{r}}=1$ signifies that the contract was terminated by a return.

A popular specification in the literature is to use a proportional hazard model for grouped data that allows a flexible baseline hazard (see Han and Hausman 1990; Meyer 1990). Let the data be divided into $M$ intervals, $\left(d_{0}, d_{1}\right],\left(d_{1}, d_{2}\right], \ldots,\left(d_{M-1}, d_{M}\right]$, where the $m$ th interval includes the duration in the $\left(d_{m-1}, d_{m}\right]$ interval. In our sample, 
we use 4-week intervals up to $d_{M}=52$ weeks; any duration beyond 52 weeks is considered censored. ${ }^{6}$ Using a proportional hazard model, the conditional probability of terminating the RTO contract in the $m$ th interval via route $j$ equals

$$
\operatorname{Pr}\left(T_{j}=m \mid X, \eta_{j} ; T>m-1\right)=1-\exp \left(-\eta_{j} \exp \left(X^{\prime} \beta_{j}+\delta_{j, m}\right)\right)
$$

$X$ is a factor vector representing customer and transaction characteristics; $\eta_{j}$ accounts for unobserved heterogeneity; given a baseline hazard $h_{0, j}, \delta_{j, m} \equiv$ $\ln \left(\int_{d_{m-1}}^{d_{m}} h_{0, j}(s) d s\right)$ is a parameter that is estimated along with $\beta_{j} ; j=r, p, s$. The joint survivor function conditional on $X$ and the unobserved heterogeneities $\eta_{\mathrm{r}}, \eta_{\mathrm{p}}$, and $\eta_{\mathrm{s}}$ is: ${ }^{7}$

$$
\begin{aligned}
S & \left(m_{\mathrm{r}}, m_{\mathrm{p}}, m_{\mathrm{S}} \mid X, \eta_{\mathrm{r}}, \eta_{\mathrm{p}}, \eta_{\mathrm{s}}\right)=\operatorname{Pr}\left(T_{\mathrm{r}}>d_{m_{\mathrm{r}}-1}, T_{\mathrm{p}}>d_{m_{\mathrm{p}}-1}, T_{\mathrm{S}}>d_{m_{\mathrm{s}}-1} \mid X, \eta_{\mathrm{r}}, \eta_{\mathrm{p}}, \eta_{\mathrm{s}}\right) \\
= & -\exp \left[\eta_{\mathrm{r}} \sum_{t=1}^{m_{\mathrm{r}}-1} \exp \left(X^{\prime} \beta_{\mathrm{r}}+\delta_{r, t}\right)+\eta_{\mathrm{p}} \sum_{t=1}^{m_{\mathrm{p}}-1} \exp \left(X^{\prime} \beta_{\mathrm{p}}+\delta_{p, t}\right)\right. \\
& \left.+\eta_{\mathrm{s}} \sum_{t=1}^{m_{\mathrm{s}}-1} \exp \left(X^{\prime} \beta_{\mathrm{S}}+\delta_{s, t}\right)\right]
\end{aligned}
$$

Due to competing risks, we observe the minimum of the latent durations along with an indicator variable that defines the mode of exit. Consider an RTO contract that terminates in the $m$ th interval, let $\Pi_{\mathrm{r}}\left(m \mid X, \eta_{\mathrm{r}}, \eta_{\mathrm{p}}, \eta_{\mathrm{s}}\right), \Pi_{\mathrm{p}}\left(m \mid X, \eta_{\mathrm{r}}, \eta_{\mathrm{p}}, \eta_{\mathrm{s}}\right)$, and $\Pi_{\mathrm{s}}\left(m \mid X, \eta_{\mathrm{r}}, \eta_{\mathrm{p}}, \eta_{\mathrm{s}}\right)$ denote the probabilities of termination via return, purchase, and skip, respectively, conditional on $\eta_{\mathrm{r}}, \eta_{\mathrm{p}}$, and $\eta_{\mathrm{s}}$. Further, define $\Pi_{\mathrm{c}}\left(m \mid X, \eta_{\mathrm{r}}, \eta_{\mathrm{p}}, \eta_{\mathrm{s}}\right)$ as the conditional probability that the RTO contract is right censored, to account for the transactions that are still active at the end of the data acquisition period. These conditional probabilities are expressed as:

$$
\begin{aligned}
\Pi_{\mathrm{r}}\left(m \mid X, \eta_{\mathrm{r}}, \eta_{\mathrm{p}}, \eta_{\mathrm{s}}\right)= & S\left(m, m, m \mid X, \eta_{\mathrm{r}}, \eta_{\mathrm{p}}, \eta_{\mathrm{s}}\right) \\
& -S\left(m+1, m, m \mid X, \eta_{\mathrm{r}}, \eta_{\mathrm{p}}, \eta_{\mathrm{s}}\right)-c c \\
\Pi_{\mathrm{p}}\left(m \mid X, \eta_{\mathrm{r}}, \eta_{\mathrm{p}}, \eta_{\mathrm{s}}\right)= & S\left(m, m, m \mid X, \eta_{\mathrm{r}}, \eta_{\mathrm{p}}, \eta_{\mathrm{s}}\right) \\
& -S\left(m, m+1, m \mid X, \eta_{\mathrm{r}}, \eta_{\mathrm{p}}, \eta_{\mathrm{s}}\right)-c c \\
\Pi_{\mathrm{s}}\left(m \mid X, \eta_{\mathrm{r}}, \eta_{\mathrm{p}}, \eta_{\mathrm{s}}\right)= & S\left(m, m, m \mid X, \eta_{\mathrm{r}}, \eta_{\mathrm{p}}, \eta_{\mathrm{s}}\right) \\
& -S\left(m, m, m+1 \mid X, \eta_{\mathrm{r}}, \eta_{\mathrm{p}}, \eta_{\mathrm{s}}\right)-c c \\
\Pi_{\mathrm{c}}\left(m \mid X, \eta_{\mathrm{r}}, \eta_{\mathrm{p}}, \eta_{\mathrm{s}}\right)= & S\left(m, m, m \mid X, \eta_{\mathrm{r}}, \eta_{\mathrm{p}}, \eta_{\mathrm{s}}\right)
\end{aligned}
$$

\footnotetext{
${ }^{6}$ Given the sporadic distribution of completed durations, especially in the skip category, we could not extend the grid beyond 52 weeks. Due to forced censoring at 52 weeks, the proportion of censored observations increased from $14.7 \%$ (reported in Table 1) to $22.6 \%$.

7 McCall (1996) and Deng et al. (2000) sum to $m_{j}$, in place of $m_{j-1}$, due to the difference in the construction of the grouped duration variable.
} 
The correction factor, $c c$, is necessary because of the discrete nature of the latent duration variables. In particular, when an event terminates in a given interval due to a particular risk, it implies that that risk's duration is less than that of any other risk; however, termination by another risk is also feasible in that same interval. Therefore, following McCall (1996), we use the following adjustment to a three risk model:

$$
c c=\frac{1}{3}\left[\begin{array}{l}
2 S\left(m, m, m X, \eta_{\mathrm{r}}, \eta_{\mathrm{p}}, \eta_{\mathrm{s}}\right)+S\left(m+1, m+1, m+1 X, \eta_{\mathrm{r}}, \eta_{\mathrm{p}}, \eta_{\mathrm{s}}\right) \\
-S\left(m+1, m, m X, \eta_{\mathrm{r}}, \eta_{\mathrm{p}}, \eta_{\mathrm{s}}\right)-S\left(m, m+1, m X, \eta_{\mathrm{r}}, \eta_{\mathrm{p}}, \eta_{\mathrm{s}}\right) \\
-S\left(m, m, m+1 X, \eta_{\mathrm{r}}, \eta_{\mathrm{p}}, \eta_{\mathrm{s}}\right)
\end{array}\right]
$$

In order to estimate the model, the distribution of the unobserved heterogeneities must be specified. An attraction of a grouped proportional hazard model is that it can easily be extended to incorporate a multi-dimensional discrete distribution for the unobserved heterogeneity, thereby allowing correlation between risks (see McCall 1996; Deng et al. 2000). We model the joint distribution of $\eta_{\mathrm{r}}, \eta_{\mathrm{p}}, \eta_{\mathrm{s}}$ by allowing $K$ distinct, though unobservable, groups in the population of RTO customers. Further, an RTO customer in group $k$ is characterized by a triplet of location parameters $\left(\eta_{r, k}, \eta_{p, k}, \eta_{s, k}\right)$ that occur with relative frequency $p_{k}$ where $k=1,2, \ldots, K$ and

$\sum_{k=1}^{K} p_{k}=1$. The location parameters and the mass points, $\varsigma_{k}$, are estimated along with other parameters of the model where $p_{k} \equiv \varsigma_{k}\left(\sum_{i=1}^{K} \varsigma_{i}\right)^{-1}$. For identification, the mass point $\varsigma_{1}$ and the shape parameter $\delta_{j, 1}$ are normalized.

For estimation, the unconditional probabilities for $j=r, p, s, c$ are expressed as:

$$
\Pi_{j}(m \mid X)=\sum_{k=1}^{K} \Pi_{j}\left(m X, \eta_{r, k}, \eta_{p, k}, \eta_{s, k}\right) p_{k}
$$

The log-likelihood function for the resulting correlated competing risk model is:

$$
\begin{aligned}
\ln \mathcal{L}= & \sum_{i=1}^{N}\left\{I_{r, i} \ln \left[\Pi_{\mathrm{r}}\left(m_{i} \mid X_{i}\right)\right]+I_{p, i} \ln \left[\Pi_{\mathrm{p}}\left(m_{i} \mid X_{i}\right)\right]+I_{s, i} \ln \left[\Pi_{\mathrm{s}}\left(m_{i} \mid X_{i}\right)\right]\right. \\
& \left.+I_{c, i} \ln \left[\Pi_{\mathrm{c}}\left(m_{i} \mid X_{i}\right)\right]\right\}
\end{aligned}
$$

The indicator variables $I_{\mathrm{r}, i}, I_{\mathrm{p}, i}$ and $I_{\mathrm{s}, i}$ equal 1 if the RTO transaction ends via return, purchase, or skip, respectively, and 0 otherwise. Similarly, $I_{\mathrm{c}, i}$ equals 1 for censored observations and 0 otherwise.

\section{Sample characteristics}

The data represent detailed transactional records from four stores of a small RTO chain in the Southeast and consist of all transactions originated between June 1, 2000 and May 31, 2002-a total of 7,517 observations after restricting attention to contracts 
lasting at least 6 months. ${ }^{8}$ Again, the dependent variable is the actual duration (length of time the contract actually ran) of an RTO contract conditional on a given exit and we consider three mutually exclusive and collectively exhaustive exits: return, purchase, and skip. We note that most customers exercise one of the embedded options-either to return or to purchase early — and so the actual running time of the contract is typically less than the contractual term that assumes all payments are made. Of the transactions in our sample, $85.3 \%$ have concluded (see Table 1), either as return (62.2\%), purchase $(20.1 \%)$, or skip $(3.0 \%)$. For the remaining $14.7 \%$ of the cases, the contract is still active, with the customer continuing to pay rent, resulting in right-censored observations. For all completed contracts, the average contract duration is 17.15 weeks with about 10 weeks for returns and 40 weeks for purchases.

It is worth pointing out that of all completed contracts, $72.9 \%$ end with the item returned and only $23.6 \%$ represent a purchase. Perhaps surprisingly, these results are at variance with the ones reported by the Federal Trade Commission (2000). The FTC study is a consumer survey, suggesting that of the transactions completed at the time of survey, $64.3 \%$ resulted in acquisition. This discrepancy can be partly reconciled by considering the data sets' emphases, i.e., survey versus transaction-based. While survey data are prone to misreporting, transaction data have some tendency to overstate return as a customer may instigate several transactions before eventual merchandise acquisition. Further, our data show a substantial amount of unrecovered merchandise which is unlikely to be reported in a survey - this is the $3.5 \%$ of completed transactions which are skips (3.0\% of total transactions).

A number of independent variables are postulated to influence how long an agreement will last and how it will terminate. These variables can be characterized as either demographic, contract structure, or repeat customer. More casually, they deal with who the customer is, what the contract looks like, and how it is used. They are detailed below. ${ }^{9}$

Seven demographic variables are considered. AGE equals 1 if more than 25 years of age (and equals 0 otherwise). GENDER is 1 if male. MARITAL STATUS is 1 if married. EMPLOYMENT equals 1 if employed for over 6 months. INCOME is a qualitative variable measured in $\$ 1,000$ s where 1 is for $0-5,2$ for $5-10,3$ for $10-15,4$ for $15-20,5$ for 20-25, and 6 for more than 25. GOVERNMENT aid equals 1 if the customer receives government aid in the form of TANF (temporary aid for needy families), social security, or welfare. REFERRAL is 1 if the customer has been referred to the store either

\footnotetext{
8 The information is gathered with the cooperation of the Association of Progressive Rental Organizations (APRO), the industry trade group; High Touch, Inc., the primary supplier of RTO store management software; and the anonymous chain owner. Regarding the underlying data, the history of an individual transaction is automatically purged 14 months after concluding. To avoid a truncation issue, attention was restricted to this 14 month period; and, to extend the available window, the data were regathered at a later period and merged. Consequently, some transactions were still on-going at time of collection and thus are censored. We have analyzed other store data over different time periods and, despite some variations across regions and between urban and rural areas, have found the basic characteristics of the data surprisingly consistent.

${ }^{9}$ Given the number of seemingly related variables, especially the demographics, care was taken to address possible multicollinearity. Among the diagnostics used, variance inflation factors were calculated; these ranged from 1.03 to 3.93 with a median of 1.61 , suggestive that the data does not suffer from serious multicollinearity.
} 


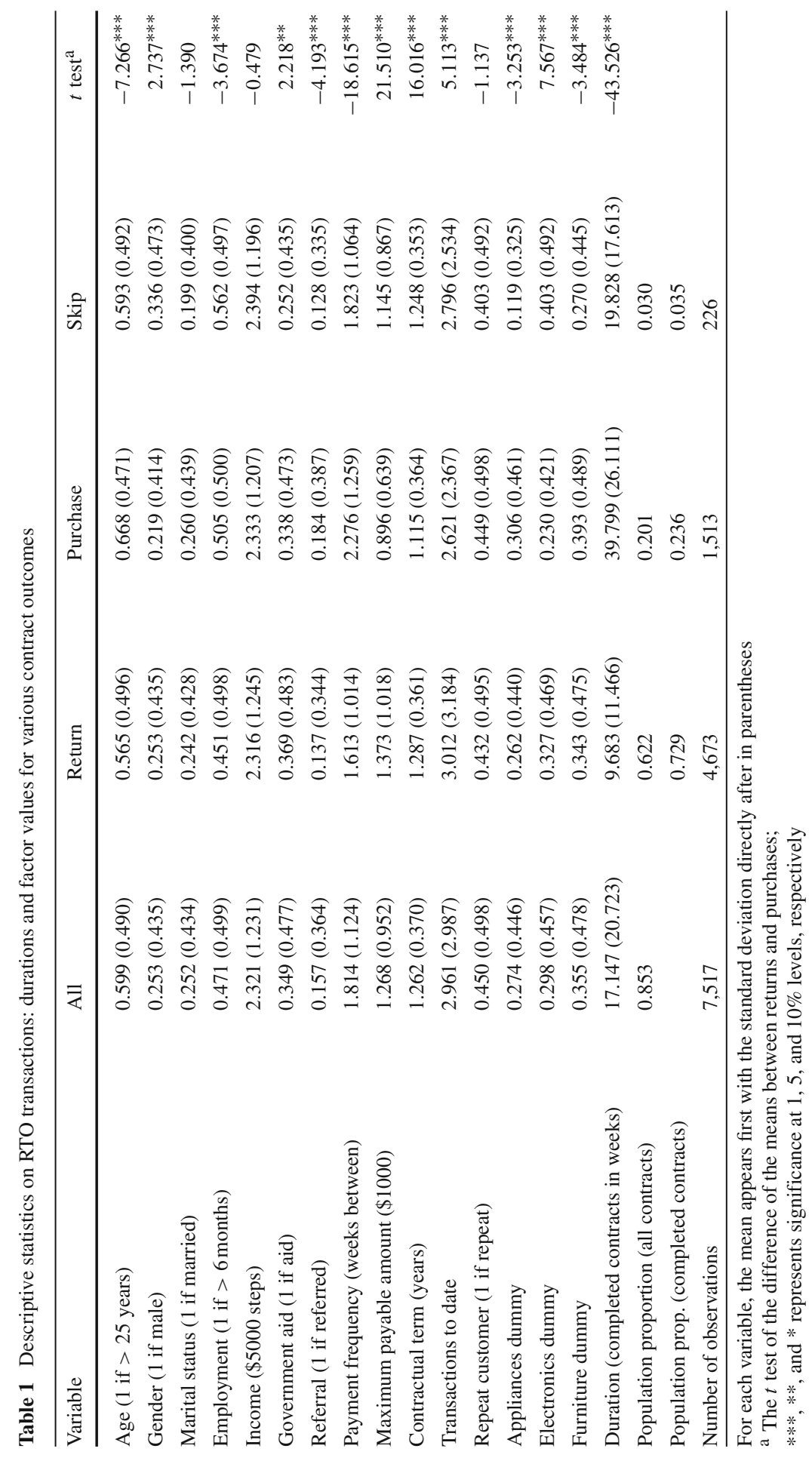


directly or by a friend or relative. Taken together, they show a customer base which is young, predominately female, unmarried, employed a fairly short period of time in their current job for low wages, a high incidence of government assistance, and with a significant amount referred (see Table 1). This picture is consistent with existing literature, e.g., Lacko et al. (2002).

Contract structure is captured with three variables. PAYMENT FREQUENCY is defined as a qualitative variable taking value 1 for weekly, 2 for biweekly, and 4 for monthly payment schedule; this metric captures the number of weeks between payments. MAXIMUM PAYABLE AMOUNT is the upper bound on rent paid and equals the periodic payment amount multiplied by the total number of payments; measured in $\$ 1,000$ s. CONTRACTUAL TERM is the maximum length in years of the agreement, if it goes full term. Notice these three variables completely capture the financial aspect of acquiring merchandise by paying to term. Namely, the payment schedule, total due, and contractual period. Roughly, the average is payment every 2 weeks, totaling $\$ 1,250$ over 15 months.

Two repeat customer variables are included. TRANSACTIONS TO DATE measures the number of transactions up to and including the agreement under consideration. REPEAT CUSTOMER is 1 if the customer has had several distinct transactions to date. As the mean transactions to date is close to 3 , multiple transactions are clearly common for RTO customers. However, there are several ways to generate multiple deals without being a true repeat-e.g., due to service or exchange—and so a dummy is included to capture those customers with unrelated transactions, representing some $45 \%$ of the data set. Importantly, both variables are defined conditional only on information actually available when a given transaction was initiated. Thus, an endogeneity issue is avoided as only chronologically prior transactions (i.e., a priori knowable information) are considered in classifying a given transaction as a "repeat" or not.

Finally, dummy variables are included for the three main merchandise classifications-APPLIANCES, ELECTRONICS, and FURNITURE. These represent 27.37, 29.77, and $35.48 \%$ of the transactions, respectively, a total of $92.62 \%$ of the data.

The means and standard deviations of the above variables are presented in Table 1, both in terms of the overall sample and for each destination. The $t$ test for the difference of means between returns and purchases is also reported. Interestingly, the characteristics of renters are quite distinct from those of the buyers, especially those dealing with contract structure. A casual look at the table also suggests that purchasers, relative to renters, are more likely to be older consumers who are employed. Renters are more likely to be on a weekly payment schedule, especially when the maximum payable amount is large. Also, among types of merchandise, electronics are more likely to be rented, while appliances and furniture are more likely to be purchased.

Much noted in the literature and in policy discussions is the high reported annual percentage rates (APRs) associated with RTO contracts. Such calculations are, of course, based on assuming that consumers make all rental payments until maturity. Our transactional database allows us insight into the actual effective rate-i.e., the rate based on the actual payments made. To illustrate, suppose an APR of 200\% (e.g., Zikmund-Fisher and Parker 1999 report a 229.7\% APR on a television) then an imputed 
periodic payment along with a markup of total rent to cost can be calculated. ${ }^{10}$ Using the average durations (length of time the contracts actually ran) and contractual terms from Table 1 along with an assumption on salvage value, average effective rates can then be determined for each destination. Consistent with Federal Trade Commission (2000), we assume an early purchase option of 50\% of the remaining payments and use $s=0.7$ as a point estimate of the salvage value for a returned item, $s=0$ for skips. In our sample, we obtained 192, 66, and $-69 \%$ for purchase, return, and write off, respectively. Further, weighting by the sample proportions from Table 1 yields an a priori effective rate of $91 \%$. While this estimate is still quite high, it is much reduced from the reported APR. This computation is just for illustration and is based on a conservative estimate of salvage value. For instance, if one assumes that the average return item retains only $60 \%$ of its retail value, the overall effective rate drops to $48 \%{ }^{11}$

\section{Results}

Table 2 presents the maximum likelihood estimates of the dependent competing risk models assuming that there are two distinct population groups of RTO customers $(K=$ $2) .{ }^{12}$ In other words, the estimated triplet of location parameters are $\left(\eta_{r 1}, \eta_{p 1}, \eta_{s 1}\right)$ for one group and $\left(\eta_{r 2}, \eta_{p 2}, \eta_{s 2}\right)$ for the other. The difference between these groups may be attributed to the unobserved intentions at the initiation of an RTO contract (e.g., predisposition to return or purchase) or other unobserved demand or income shocks.

We start with a brief analysis of the raw (Kaplan-Meier) conditional termination rates for RTO transactions. For a given route, these raw termination rates are computed, for each of the $M$ intervals defined above, as:

$$
h_{j}^{*}(m)=\frac{N_{j}(m)}{\left(d_{m}-d_{m-1}\right)\left(N_{j}(m)+N_{n}(m)\right)},
$$

where $N_{j}(m)$ represents the number of exits in $\left(d_{m-1}, d_{m}\right]$ to route $j$ and $N_{n}(m)$ is the number of uncensored transactions that are not terminated by $d_{m}$. Further, we estimate the overall conditional termination rates for the competing risk model, evaluated at average factor values $\bar{X}$, as:

\footnotetext{
10 Norming the item's cost to $\$ 1$ and letting $p$ be the monthly payment due, $d$ the contract's actual duration, and $s$ the salvage value, the effective interest rate is $12 i$ where $i$ solves $p\left[1-(1+i)^{-d}\right] i^{-1}+s(1+i)^{-d}=$ 1. While the calculation is conceptually simple, note that the APR of a rental is dependent on the item's estimated drop in value over the rental period, $(1-s)$, and that the amount of depreciation would vary across merchandise.

11 We discuss APR as it is an important part of the policy debate. We feel, however, that the concept is deficient as a cost metric for RTO. The embedded options along with the possibility of financial reversal among the clientele lead to a very rich set of possible contract evolutions. Consequently, there is likely to be significant variance between actual use and the assumed "typical use" employed to calculate APR-thus, the true effective cost could be quite different and vary from case to case.

12 We sought to extend the analysis to three groups $(K=3)$ however, the location and mass point parameters were insignificant; further, the likelihood ratio test corroborated that the two group model was more appropriate.
} 
Table 2 Estimates of the competing risk model with unobserved heterogeneity

\begin{tabular}{|c|c|c|c|}
\hline Parameter & Return & Purchase & Skip \\
\hline Age $(1$ if $>25$ years $)$ & $-0.249^{* * *}(-4.560)$ & $-0.138(-1.387)$ & $-0.465^{* * *}(-2.834)$ \\
\hline Gender ( 1 if male) & $0.098 *(1.857)$ & $-0.143(-1.445)$ & $0.205(1.342)$ \\
\hline Marital status (1 if married) & $-0.046(-0.839)$ & $0.044(0.471)$ & $-0.362^{* *}(-2.125)$ \\
\hline Employment ( 1 if $>6$ months $)$ & $-0.202^{* * *}(-3.435)$ & $-0.010(-0.093)$ & $-0.012(-0.072)$ \\
\hline Income & $0.139 * * *(5.807)$ & $0.048(1.139)$ & $0.016(0.215)$ \\
\hline Government aid ( 1 if aid) & $0.341^{* * *}(5.324)$ & $0.124(1.083)$ & $-0.467^{* *}(-2.420)$ \\
\hline Referral (1 if referred) & $-0.301^{* * *}(-5.033)$ & $0.020(0.189)$ & $-0.578^{* * *}(-2.732)$ \\
\hline $\begin{array}{l}\text { Payment frequency } \\
\text { (weeks between) }\end{array}$ & $-0.613^{* * *}(-24.363)$ & $0.065(1.451)$ & $-0.440^{* * *}(-4.783)$ \\
\hline Maximum payable amount & $0.218 * * *(8.256)$ & $-0.517^{* * *}(-6.173)$ & $0.024(0.233)$ \\
\hline Contractual term & $-0.510^{* * *}(-7.034)$ & $-3.580^{* * *}(-24.612)$ & $-1.327^{* * *}(-5.415)$ \\
\hline Transactions to date & $0.047 * * *(5.014)$ & $-0.007(-0.366)$ & $0.052 *(1.837)$ \\
\hline Repeat customer ( 1 if repeat) & $-0.336^{* * *}(-6.281)$ & $-0.064(-0.675)$ & $-0.719^{* * *}(-4.286)$ \\
\hline Appliances dummy & $0.059(0.657)$ & $0.300 * *(2.120)$ & $-1.465^{* * *}(-6.535)$ \\
\hline Electronics dummy & $0.318 * * *(3.571)$ & $0.163(1.140)$ & $-0.291(-1.596)$ \\
\hline Furniture dummy & $-0.110(-1.237)$ & $0.476 * * *(3.555)$ & $-1.097^{* * *}(-5.695)$ \\
\hline$\eta_{1}$ (location parameter) & $4.138 * * *(6.829)$ & $2.838 * * *(3.489)$ & $1.186^{* *}(2.281)$ \\
\hline$\eta_{2}$ (location parameter) & $0.482 * * *(6.321)$ & $1.881 * * *(3.880)$ & $0.260 * *(2.473)$ \\
\hline$\varsigma_{2}($ mass point $)$ & $0.787 * * *(10.553)$ & & \\
\hline
\end{tabular}

$t$ statistics are in parentheses; $* * *, * *$, and $*$ represents significance at 1,5 , and $10 \%$, respectively; $\varsigma_{1}$ is normalized to 1.0 in estimation; the shape parameters $\delta_{j, m}$, with $\delta_{j, 1}$ normalized, are not reported

$$
h_{j}(m \mid \bar{X})=\frac{{ }_{k=1}^{K} \Pi_{j}\left(m \mid \bar{X}, \hat{\eta}_{r k}, \hat{\eta}_{p k}, \hat{\eta}_{s k}\right) \hat{p}_{k}}{\left(d_{m}-d_{m-1}\right){ }_{k=1}^{K} S\left(m, m, m \mid \bar{X}, \hat{\eta}_{r k}, \hat{\eta}_{p k}, \hat{\eta}_{s k}\right) \hat{p}_{k}} .
$$

We also compute analogous rates for each of these two distinct groups of RTO customers using $\left(\hat{\eta}_{r 1}, \hat{\eta}_{p 1}, \hat{\eta}_{s 1}\right)$ and $\left(\hat{\eta}_{r 2}, \hat{\eta}_{p 2}, \hat{\eta}_{s 2}\right)$, respectively, rather than averaging them out as above.

The estimated conditional termination rates are presented in Fig. 1. For return, purchase, and skip, these rates are generally decreasing, increasing, and constant, respectively. Further, casual observation suggests the basic pattern of the raw termination rates resemble those of the overall conditional termination rates quite well. The figure also illustrates that the overall rate masks very different behavior among the two distinct groups of RTO customers; 56\% of customers $(=1 /(1+0.787))$ fall in group one, the remaining $44 \%$ into group two. Note that $\hat{\eta}_{j 1}>\hat{\eta}_{j 2}$ for all $j=r, p, s$ suggesting that group one contracts tend to conclude faster. Furthermore, as this ratio is significantly greater for $j=r$ than for $j=p, s$, the magnitude of the difference between the groups is starker for contracts concluding with merchandise return. We will explore these group differences in greater detail later.

We now turn to the estimates of the competing risk model (Table 2). Most variables appear to be statistically significant in explaining the conditional probability of an 

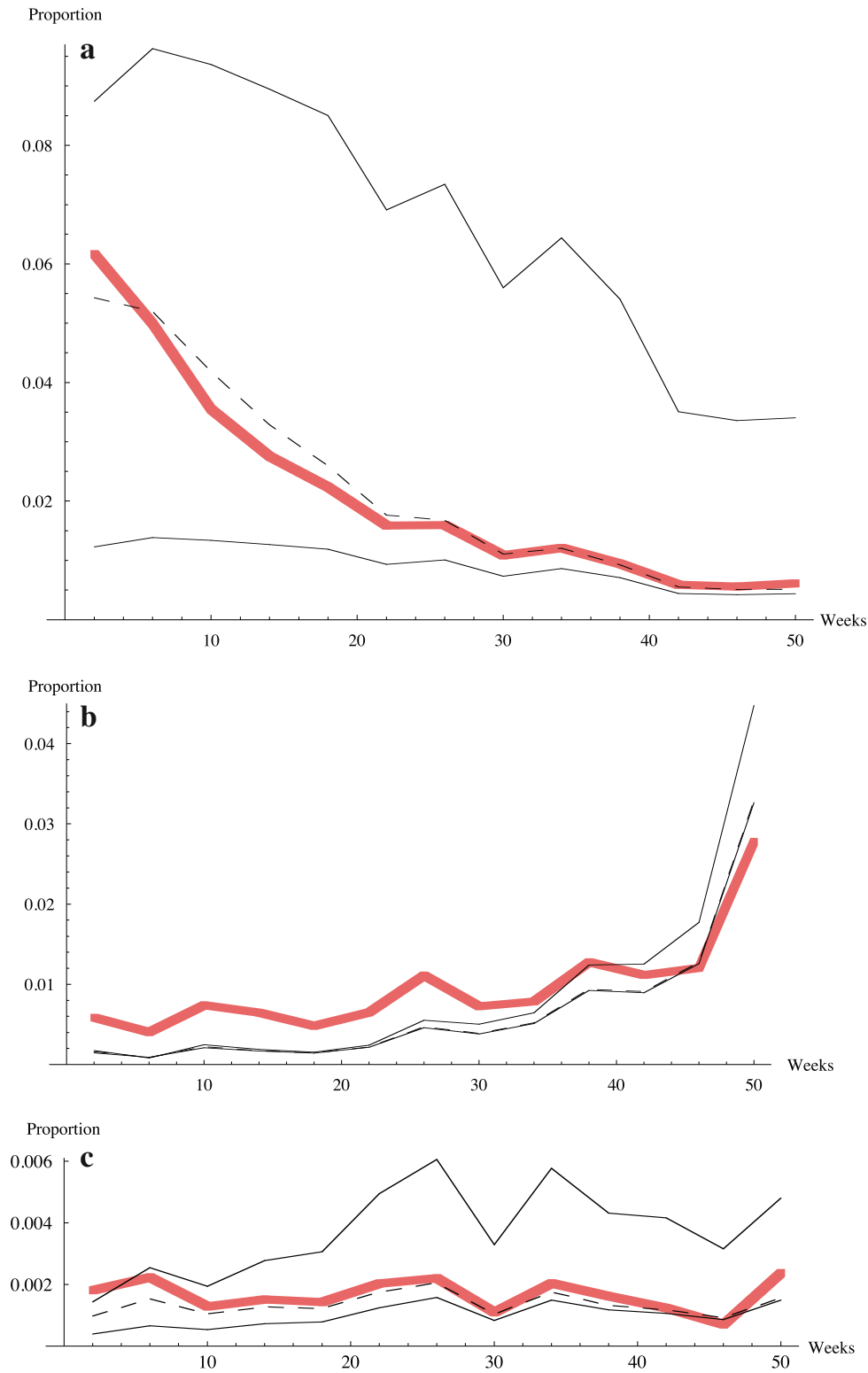

Fig. 1 Raw and estimated conditional termination rates. a Return rate, top (bottom) solid line is group 1(2), overall is dashed; heavy line is raw rate. b Purchase rate, top (bottom) solid line is group 1(2), overall is dashed; heavy line is raw rate. c Skip rate. The figure compares the raw conditional termination rate (thick line) with that estimated by the model. The top, middle, and bottom panes present the return, purchase, and skip exits, respectively. In each pane, the highest to lowest lines represent the exit proportions for group 1, overall, and group 2, respectively; for added emphasis, a dashed line is used for the overall group. Comparing the overall estimate to that of the raw illustrates the general goodness of fit. Note for the purchase exit the three estimated lines are nearly coincident, with group 1 having a noticeably higher probability for the latter part of the time interval 
RTO contract terminating via various exit routes; indeed all variables are significant at the $10 \%$ level for at least one exit and all but two are significant at $1 \%$. In general, the demographic variables appear to have lower explanatory power relative to the other more transaction-specific variables. Also noteworthy is the highly significant nature of the contract structure variables.

There is a known problem of interpreting regression coefficients in competing risk models as it is not transparent how changes in a factor influence the probability of exit via a given route (see Thomas 1996). In general, one has to look at the relative magnitude of the coefficients for all exits to draw a conclusive picture. The problem is further exacerbated by the incorporation of unobserved heterogeneity which allows correlation between risks. In order to illustrate the influence of each factor, we estimate the overall unconditional probability of exit $\Pi_{j}(X)$ for $j=r, p, s$ evaluating it at the various factor values. We compute this probability as:

$$
\Pi_{j}(X)=\sum_{m=1}^{M+1} \Pi_{j}(m \mid X)
$$

$\Pi_{j}(m \mid X)$ is estimated from (8) for $m=1,2, \ldots, M$; for the " $>52$ " interval, we distribute $\Pi_{\mathrm{c}}(M+1 \mid X)$ among the three risk routes on the basis of the relative magnitude of $\Pi_{j}(M \mid X)$. We also compute expected duration, conditional on exit via route $j$, as:

$$
E_{j}(X)=\Pi_{j}^{-1} \sum_{m=1}^{M+1} m_{d} \Pi_{j}(m \mid X)
$$

where $m_{d}$ is the mid-point of the class interval; for the " $>52$ " interval, we use 72 weeks as a mid-point.

When all factors are evaluated at their mean levels, the unconditional probabilities of return, purchase, and loss are computed as $0.713,0.250$, and 0.036 , respectively; with corresponding expected contract durations of $12.49,57.10$, and 30.11 weeks. In general, these values seem to match the data very well-see Table 1 for comparison. Further, Tables 3 and 4 compute the unconditional probability and corresponding expected duration for each factor at its 10th and 90th percentile values holding all other factors at their respective mean values. The information on expected durations supplements that on unconditional probabilities by estimating how long the contract is expected to last if it concludes via a particular exit. Hence, it provides information on how long an item was on rent, at what point the early purchase option was exercised, or when merchandise under agreement was written off as unrecoverable.

Taken together, these simulation tables resolve the ambiguity of parameter evaluation and allow some interesting observations. The relative impact of the demographic variables is generally weak in magnitude. Older (over 25) customers who are employed at least 6 months at their current job but at low wages are more likely to be purchasers and generally have a longer duration of an RTO contract regardless of exit. This is consistent with the notion that a key demographic for RTO is the "working poor." That is, the older one gets, still employed at low wages, the fewer financial options one has 
Table 3 Return, purchase, and loss probabilities evaluated at various factor values

\begin{tabular}{|c|c|c|c|c|}
\hline \multirow[t]{2}{*}{ Variable } & \multicolumn{4}{|c|}{$10^{\text {th }}$ Percentile ( $90^{\text {th }}$ Percentile) } \\
\hline & Value & Return & Purchase & Skip \\
\hline Age ( 1 if $>25$ years $)$ & $0.000(1.000)$ & $0.732(0.701)$ & $0.226(0.266)$ & $0.042(0.033)$ \\
\hline Gender ( 1 if male) & $0.000(1.000)$ & $0.708(0.729)$ & $0.257(0.230)$ & $0.035(0.042)$ \\
\hline Marital status (1 if married) & $0.000(1.000)$ & $0.714(0.710)$ & $0.246(0.263)$ & $0.040(0.028)$ \\
\hline Employment ( 1 if > 6 months) & $0.000(1.000)$ & $0.730(0.694)$ & $0.236(0.266)$ & $0.034(0.040)$ \\
\hline Income & $1.000(4.000)$ & $0.682(0.751)$ & $0.275(0.219)$ & $0.043(0.030)$ \\
\hline Government aid ( 1 if aid) & $0.000(1.000)$ & $0.690(0.753)$ & $0.262(0.226)$ & $0.049(0.021)$ \\
\hline Referral (1 if referred) & $0.000(1.000)$ & $0.720(0.673)$ & $0.241(0.301)$ & $0.039(0.027)$ \\
\hline Payment frequency (weeks between) & $1.000(4.000)$ & $0.798(0.421)$ & $0.166(0.544)$ & $0.035(0.035)$ \\
\hline Maximum payable amount & $0.381(2.495)$ & $0.656(0.789)$ & $0.308(0.173)$ & $0.035(0.038)$ \\
\hline Contractual term & $0.753(1.552)$ & $0.661(0.749)$ & $0.311(0.209)$ & $0.028(0.042)$ \\
\hline Transactions to date & $1.000(6.000)$ & $0.697(0.737)$ & $0.267(0.224)$ & $0.035(0.038)$ \\
\hline Repeat customer ( 1 if repeat) & $0.000(1.000)$ & $0.733(0.687)$ & $0.222(0.285)$ & $0.045(0.028)$ \\
\hline Appliances dummy & $0.000(1.000)$ & $0.705(0.719)$ & $0.237(0.262)$ & $0.057(0.019)$ \\
\hline Electronics dummy & $0.000(1.000)$ & $0.696(0.751)$ & $0.260(0.192)$ & $0.044(0.057)$ \\
\hline Furniture dummy & $0.000(1.000)$ & $0.720(0.675)$ & $0.224(0.294)$ & $0.056(0.031)$ \\
\hline
\end{tabular}

Table 4 Expected durations evaluated at various factor values

\begin{tabular}{|c|c|c|c|c|}
\hline \multirow[t]{2}{*}{ Variable } & \multicolumn{4}{|c|}{ 10th Percentile (90th Percentile) } \\
\hline & Value & Return & Purchase & Skip \\
\hline Age ( 1 if $>25$ years $)$ & $0.00(1.00)$ & $11.554(13.194)$ & $55.974(57.774)$ & $28.664(31.078)$ \\
\hline Gender ( 1 if male) & $0.00(1.00)$ & $12.530(12.383)$ & $56.754(58.060)$ & 29.835 (30.899) \\
\hline Marital Status (1 if married) & $0.00(1.00)$ & $12.428(12.666)$ & $57.180(56.845)$ & $30.149(29.915)$ \\
\hline Employment ( 1 if $>6$ months $)$ & $0.00(1.00)$ & $12.069(13.004)$ & $56.948(57.228)$ & $29.752(30.530)$ \\
\hline Income & $1.00(4.00)$ & $13.560(11.348)$ & $57.849(55.914)$ & $31.427(28.533)$ \\
\hline Government aid ( 1 if aid) & $0.00(1.00)$ & $13.077(11.484)$ & $57.570(55.994)$ & $30.878(28.581)$ \\
\hline Referral ( 1 if referred) & $0.00(1.00)$ & $12.254(13.903)$ & $57.064(57.175)$ & $29.952(30.824)$ \\
\hline Payment frequency (weeks between) & $1.00(4.00)$ & $10.677(19.322)$ & $56.570(56.444)$ & $28.608(34.604)$ \\
\hline Maximum payable amount & $0.38(2.50)$ & $12.248(13.137)$ & $51.794(62.077)$ & $26.284(35.753)$ \\
\hline Contractual term & $0.75(1.55)$ & $8.276(17.079)$ & $29.139(64.746)$ & $17.170(41.909)$ \\
\hline Transactions to date & $1.00(6.00)$ & $12.926(11.880)$ & $57.048(57.119)$ & $30.307(29.794)$ \\
\hline Repeat customer ( 1 if repeat) & $0.00(1.00)$ & $11.651(13.656)$ & $56.541(57.660)$ & $29.154(31.157)$ \\
\hline Appliances dummy & $0.00(1.00)$ & $12.560(12.749)$ & $57.826(57.218)$ & $30.720(30.059)$ \\
\hline Electronics dummy & $0.00(1.00)$ & $13.018(11.555)$ & $57.634(58.009)$ & $30.895(30.192)$ \\
\hline Furniture dummy & $0.00(1.00)$ & $12.557(12.970)$ & $58.622(55.347)$ & 31.435 (28.948) \\
\hline
\end{tabular}

These expected durations are conditional on route-specific exit and are evaluated at the 10th and 90th percentile value of each variable, with others at their mean values. With all factors at their mean value, expected durations of a return, purchase, and skip are $12.49,57.10$, and 30.11 , respectively 
and so the more valuable RTO is as a mechanism to acquire needed goods (see also Zikmund-Fisher and Parker 1999). Also intriguing, the probability of a skip is notably higher for a young male.

As one might expect, for a repeat customer the expected duration is longer for all exits and the probability of purchase is higher, while those for return and skip are smaller. This might simply reflect satisfaction over the earlier transaction(s). Alternatively, it may be that a customer having a continuing need for RTO is likely to be more reliant on this method of acquisition. Interestingly, the transactions to date variable has the exact opposite effect on exit probabilities, probably reflecting the effect of customers exercising the embedded option of merchandise exchange. For example, a good may be returned in order to exchange it for a similar item-e.g., a customer renting a TV then returning it and, nearly simultaneously, renting a different TV, possibly repeating such an exchange several times. This represents an important consumer option to resolve uncertainty over a good's utility without having to suffer "buyer's remorse."

One key result concerns the payment frequency variable. Going from a weekly to a monthly schedule, the overall return probability almost halves (going from 0.798 to 0.421 ), while the purchase probability triples $(0.166$ to 0.544$)$. Arguably there is preselection of payment schedules by customers. In other words, those expecting to rent prefer a weekly arrangement for the greater flexibility offered, while those expecting to purchase prefer monthly for the greater convenience. We believe that pre-selection alone may not fully account for this periodicity-related effect. First, the demographic variables included in the analysis partially control for the pre-selection effect since they are likely to be correlated with the borrower's intentions. Second, as noted above, group one seems to have much more of a rental motive than does group two and yet the same qualitative pattern is observed, going from weekly to monthly, for both groups of customers (see Table 5, discussed below).

We conjecture that the above result also has a behavioral component, although it cannot be isolated from the regression results. A standard economic model would predict that consumers finance purchases so that, for a given discount rate, the present value of payments is minimized. Prelec and Loewenstein (1998) argue that the reality of consumer hedonics is quite different. When people finance their purchases, they experience a pain that can undermine the pleasure derived from consumption. They argue that the pain of paying plays an important role in self-regulation and propose a mental accounting model that incorporates the interactions between the pleasures of consumption and the pain of paying. Using a concept of coupling, which refers to the degree by which consumption reminds the consumer of payment, they argue that financing methods such as credit cards have a much weaker coupling as opposed to cash payments. In a similar vein, we believe that periodicity of payments exerts a strong influence on coupling and is important in consumer decision-making. Consumers tend to evaluate their decisions at discrete points in time and the perceived cost associated with making a payment is somewhat reduced when the evaluation points are spread out.

The contractual term variable, despite having a $1 \%$ significant negative coefficient for every exit, implies that longer contracts increase (decrease) the probability of return (purchase) and result in a greater probability of a loss. Consistent with Table 2, 


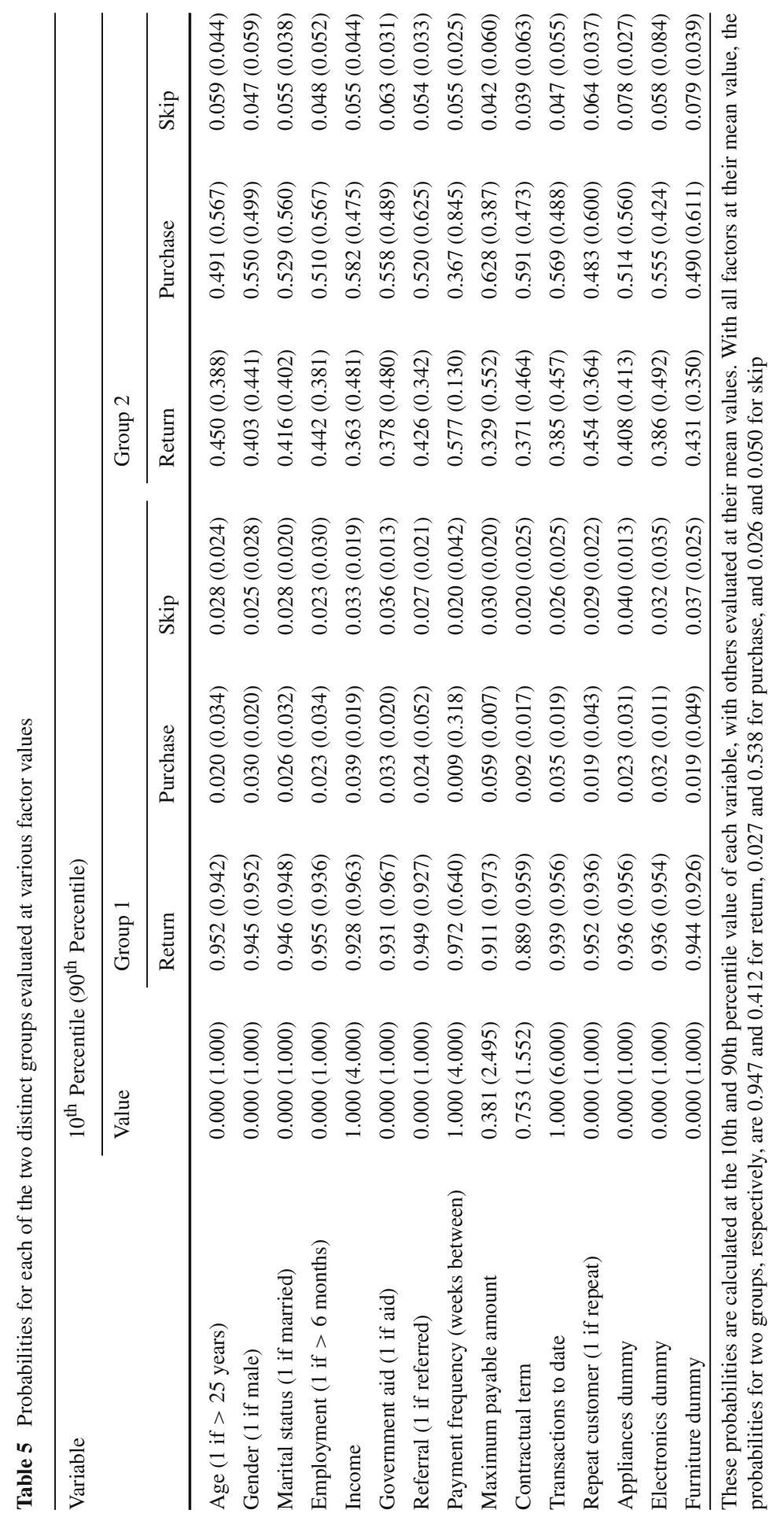


expected durations increase with contractual term for all exits. This could be viewed as saying simply that acquisition is less likely the longer the contractual term, even though the contract may still run a long time. Comparing the changes in expected duration in Table 4 shows an interesting phenomenon. As the contractual term varies between 0.75 years ( 39 weeks) and 1.55 years ( 80.6 weeks), the expected durations of the contract change proportionately for all exits. For instance, for a return, the ratio of expected duration to contractual term equals $21 \%$ whether evaluated at their 10th percentile values $(8.28 / 39.0=0.212)$ or at their 90th $(17.08 / 80.6=0.212)$. Although somewhat simplistic, this analysis makes a compelling case that consumers adjust their decision time scale to account for differences in contractual term, perhaps due to time-varying discount factors used in valuing payments (see Frederick et al. 2002). Alternatively, consumers evaluate the options embedded in these agreements and that such options have a natural exercise point.

We also note that for the electronics dummy there is a higher probability of return or skip and a lower probability of purchase. This is consistent with electronics items being viewed as luxury goods as well as the notion consumers are using RTO to acquire essential household items, namely furniture and appliances. Support for this view comes from the increase in the probability of purchase when the item is either an appliance or furniture as well as relatively lower probabilities of return and skip.

The results on unconditional probabilities and expected durations in Tables 3 and 4 can be used to gain insight into the financing cost associated with RTO agreements. They illustrate the trade-offs and cross-subsidization implicit in the RTO contractual arrangement. It is noteworthy that skips, returns, and early purchasers are subsidized by those paying to term. For a given contract, the periodic payment, which is proportional to the store's mark-up, is influenced by the purchase, return, and skip probabilities and their corresponding expected durations. Further, by conditioning these estimates on the various values of the demographic, contract structure, and repeat customer variables, arguably more realistic expected annual percentage rates (APRs) can be calculated using the procedure described in footnote 10 . With high return probabilities and with expected durations considerably shorter than the corresponding contractual terms, the estimated APR are much less than extant APR estimates which assume payments made to term (e.g., Freedman 1993). Further, we can show that the APRs computed for weekly contracts, for example, are considerably smaller than their monthly counterparts. Similar subgroup cost comparisons can also be made. For example, APRs on electronics, as a class, can be shown to be less than those on appliances which are less than those on furniture. In the case of electronics, this is driven by relatively high probabilities of return and skip as well as generally shorter durations.

Tables 5 and 6 present unconditional probabilities and expected durations for the two distinct groups of RTO customers. At first pass, group one appears to be composed of pure renters as there is a $94.7 \%$ chance of return and nearly equal probabilities of purchase (2.7\%) and skip (2.6\%) -i.e., they seem to have a well-defined short-term need and so renting is more cost-effective than purchase. In contrast, for group two, the majority of transactions are expected to lead to purchase (53.8\%), while return occurs $41.2 \%$ of the time and the skip rate, at $5 \%$, is nearly twice that for group one. A possible interpretation of group two members is that they are financially constrained with an income stream that is low mean/high variance, i.e., they represent one's a 
priori notion of the typical RTO customer. Such an income distribution would serve as a catalyst for a change in circumstance either positive-leading to a purchase or return, depending on the early purchase option's value-or negative-necessitating an involuntary return or even a skip, thereby accounting for their relatively higher purchase and skip rates. The above interpretation is supported by the expected durations in Table 6 which show, regardless of exit, deals conclude much faster for group one (group two contracts take at least 2.5 times as long to conclude). In general, group one customers seem to value the embedded put option, i.e., the right to terminate the transaction at any point, returning the item without adverse financial consequence. In contrast, group two are purchasers valuing the embedded call-which lets them convert their rental agreement into the right to own. These differences in contract usage lead to significant cross-subsidization of groups. Using the same assumptions as in Sect. 3-which lead to an overall APR of 91\%-group one's APR is estimated at $33 \%$, while group two faces an APR of $185 \%$.

To gain additional insights, Fig. 2 presents the conditional termination rates for return under two scenarios: a contract requiring weekly payments having a relatively long duration, and one with monthly payments and a short duration. Rationality suggests that the former contractual terms would be preferred by a renter to maximize flexibility and minimize rental costs, while the latter would be preferred by a purchaser to maximize convenience. At the same time, as discussed earlier, subsequent consumer behavior is also influenced by contract structure. Focusing on the overall rate, the figure shows that the monthly/short customer has a relatively low termination rate that declines by an order of magnitude over the period—going from 2 to $0.2 \%$. In contrast, the weekly/long customer probability of return starts 3.5 times larger and while it declines faster early on, it continues to be well above the monthly/short rate. Arguably pre-selection is likely to be dominant early in the contract whereas any behavioral effect would gradually build. The narrowing of the distance between the termination rates under two scenarios is perhaps indicative of a reduced pre-selection effect. However, the persistence of a significant difference is, we believe, due to behavioral reasons. Also interesting is the variation between groups. Group one return rates are highest, regardless of scenario, and focusing on the weekly/short contract reinforces the notion that customers in group one are renters, as their return probabilities are, on average, five times that of the overall rate. It also highlights the magnitude of the influence of the unobserved factors since the group one return probability under a monthly/short contract dominates that of group two's probability under a weekly/long contract over the entire interval.

\section{Conclusion}

This article provides a comprehensive multiple destination analysis for RTO contracts. A competing risk methodology is employed that accounts for unobserved heterogeneity among borrowers and estimates this simultaneously with flexible baseline hazards associated with the possible exits. In particular, we analyze the return, purchase, and skip probabilities, thus providing useful information on the characteristics of renters and purchasers. 


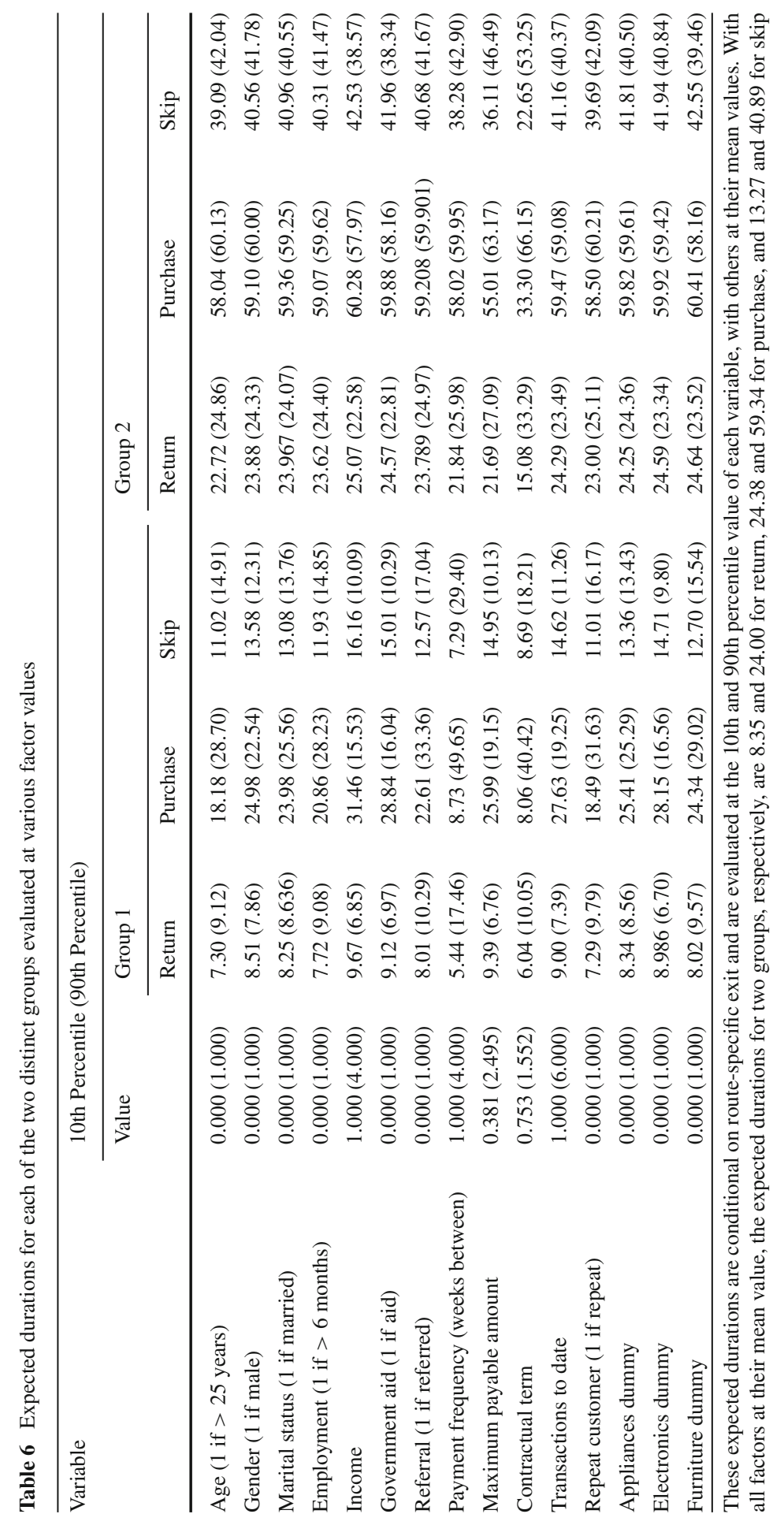




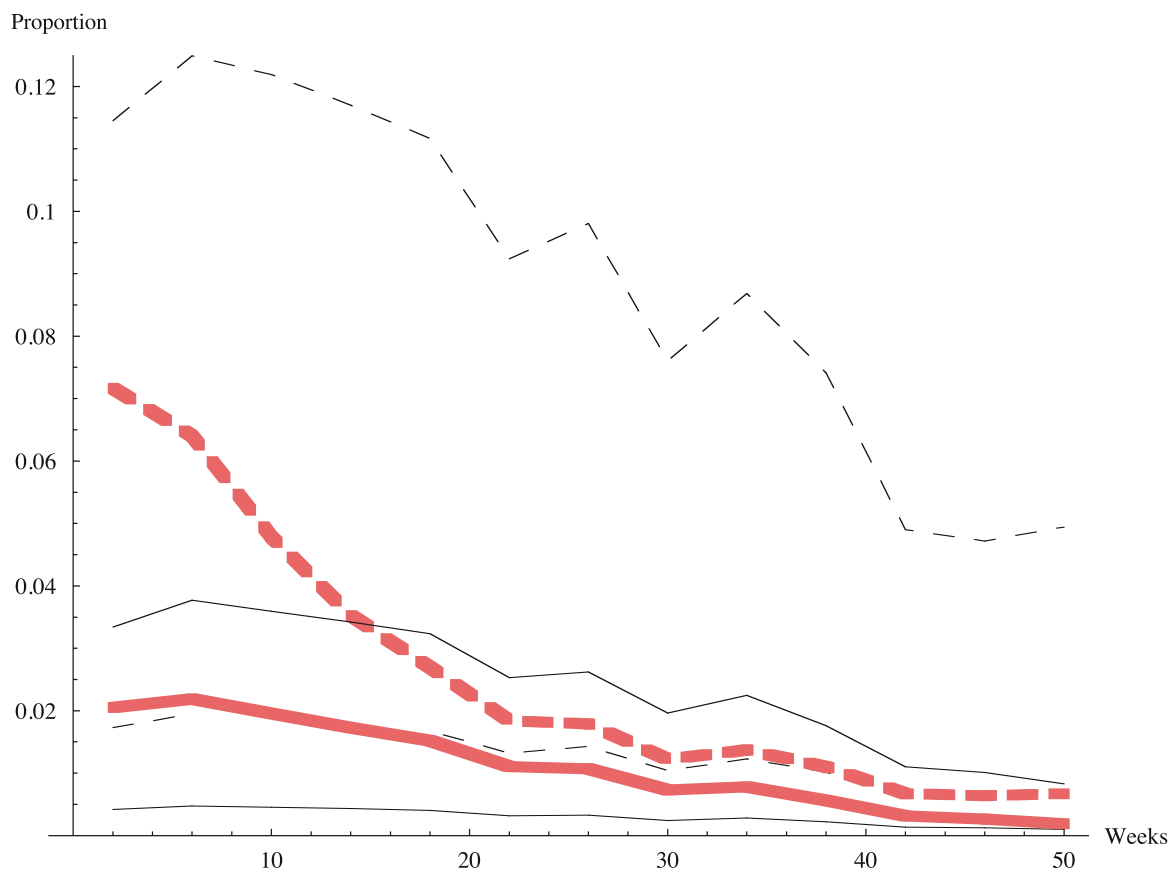

Fig. 2 Estimated termination rates of return under two scenarios. The figure contrasts two scenarios: (a) weekly payment schedule and relatively long contract length (dashed lines) with (b) monthly schedule and short length (solid lines). Within each scenario, the highest to lowest lines represent group 1, overall, and group 2, respectively; to emphasize the overall lines they are drawn thick

Our results support the notion that that RTO represents an important means of acquisition for the "working poor." For example, older consumers on the current job at least 6 months but at low wages have a higher probability of purchase and, regardless of outcome, a longer expected contract duration. In general, however, we find that the demographic variables have weak explanatory power on contract outcomes relative to the contract structure variables. For instance, payment schedules-weekly, biweekly, or monthly_have a very strong effect on contract outcome and duration. Contrasting weekly to monthly payment schedules, we find the former more likely to result in return and to conclude faster. While there is a pre-selection explanation for the contract structure variables, we believe that these results also fit the general notion that consumers mentally track the cost and benefits of a transaction with a symbolic linkage between consumption and the way the purchase is financed (see, e.g., Prelec and Loewenstein 1998; Thaler 1985). Finally, there is interesting variation across types of merchandise with electronics more likely to be rented, and appliances and furniture more likely to be purchased.

The unobserved heterogeneity is also found to be quite important in accounting for customer behavior. The model allows two distinct population groups of RTO customers with differing motives for entering a transaction. As a rough characterization, we find that the population is nearly equally split between pure renters and those 
seemingly dependent on RTO as a possible means of acquisition. Further, for a given consumer, group membership has a significant effect on contract usage.

We believe that our rational and behavioral explanations of consumer behavior in the RTO market can be generalized to other consumer loan markets. For instance, the results of our article may have general implications for linkage of contract structure with the consumer handling of debt instruments. Also, some of the our demographic insights may map in a more general setting. Further, since consumers are expected to have varying motivations, both observed and unobserved, behind their participation in any contractual relationship, this needs to be accounted for in the initial contract design and pricing.

RTO agreements have recently received considerable public attention. Several articles in the extant literature using somewhat unreliable survey data imply that most RTO consumers are potential purchasers of goods at usurious interest rates. However, the high incidence of returns in our transactions data seems to belie the prevailing interpretation, by some, of RTO customers as uninformed or unsophisticated. The results also illustrate the trade-offs and cross-subsidization implicit in the RTO contractual arrangement. It is noteworthy that skips and early-purchasers are alike in that both are subsidized by those who pay to term. This is analogous to the credit card market where both defaulters and those not incurring interest charges are being subsidized by those making only minimum payments. We compute effective interest rates paid by RTO customers which are significantly smaller than the generally reported APRs.

Acknowledgments We would like to thank Darlene Chisholm, Haldun Evrenk, Ray Jackson, and Satish Thosar and two anonymous referees for useful comments and Brian McCall both for his comments and for sharing his Fortran code with us. Any errors are ours alone.

\section{References}

Anderson MH, Jackson R (2001) A reconsideration of rent-to-own. J Consum Aff 35:295-307

Anderson MH, Jaggia S (2009) Rent-to-own agreements: customer characteristics and contract outcomes. J Econ Bus 61(1):51-69

Association of Progressive Rental Organizations (APRO) (2001) Industry revenue exceeds \$5 billion. View (APRO Newsletter), September 4

Blank R (1989) Analyzing the length of welfare spells. J Public Econ 39:245-273

Deng Y, Quigley JM, Order RV (2000) Mortgage terminations, heterogeneity and the exercise of mortgage options. Econometrica 68(2):273-307

Federal Deposit Insurance Corporation (2005) Guidelines for payday lending. www.fdic.gov/regulations/ safety/payday/

Federal Trade Commission (2000) Survey of rent-to-own customers. Bureau of Economics Staff Report, Washington, DC

Frederick S, Loewenstein G, O’Donoghue T (2002) Time discounting and time preference: a critical review. J Econ Lit 40:351-401

Freedman A (1993) Peddling dreams: a marketing giant uses its sales prowess to profit on poverty. Wall Str J A 1:A10

Han A, Hausman J (1990) Flexible parametric estimation of duration and competing risks models. J Appl Econ 5:1-28

Hill R, Ramp D, Silver L (1998) The rent-to-own industry and pricing disclosure tactics. J Public Policy Mark 17:3-10

Katz L, Meyer B (1990) Unemployment duration, recall expectations, and unemployment outcomes. Q J Econ 105:973-1002 
Lacko J, McKernan S, Hastak M (2002) Customer experience with rent-to-own transactions. J Public Policy Mark 21:126-138

Lancaster T (1990) The econometric analysis of transition data. Cambridge University Press, Cambridge

McCall BP (1996) Unemployment insurance rules, joblessness, and part-time work. Econometrica 64: $647-682$

McKernan S, Lacko J, Hastak M (2003) Empirical evidence on the determinants of rent-to-own use and purchase behavior. Econ Dev Q 17:33-52

Meyer BD (1990) Unemployment insurance and unemployment spells. Econometrica 58:757-782

Prelec D, Loewenstein G (1998) The red and the black: mental accounting of savings and debt. Mark Sci 17:4-24

Stegman MA, Faris R (2003) Payday lending: a business model that encourages chronic borrowing. Econ Dev Q 17(1):8-32

Swagler R, Wheeler P (1989) Rental-purchase agreements: a preliminary investigation of consumer attitudes and behaviors. J Consum Aff 23:145-160

Thaler R (1985) Mental accounting and consumer choice. Market Sci 4:199-214

Thomas J (1996) On the interpretation of the covariate estimates in the independent competing-risks models. Bull Econ Res 48:27-39

Walden M (1990) The economics of rent-to-own contracts. J Consum Aff 24:326-337

Zikmund-Fisher B, Parker A (1999) Demand for rent-to-own contracts: a behavioral economic explanation. J Econ Behav Organ 38:199-216 